\title{
Frequent somatic transfer of mitochondrial DNA into the nuclear genome of human cancer cells
}

\author{
Young Seok Ju, ${ }^{1}$ Jose M.C. Tubio, ${ }^{1,45}$ William Mifsud, ${ }^{1,45}$ Beiyuan Fu, ${ }^{2}$ Helen R. Davies, ${ }^{1}$ \\ Manasa Ramakrishna, ${ }^{1}$ Yilong Li, ${ }^{1}$ Lucy Yates, ${ }^{1}$ Gunes Gundem, ${ }^{1}$ Patrick S. Tarpey, ${ }^{1}$ \\ Sam Behjati, ${ }^{1}$ Elli Papaemmanuil, ${ }^{1}$ Sancha Martin, ${ }^{1}$ Anthony Fullam, ${ }^{1}$ \\ Moritz Gerstung, ${ }^{1}$ ICGC Prostate Cancer Working Group, ${ }^{46}$ ICGC Bone Cancer \\ Working Group, ${ }^{46}$ ICGC Breast Cancer Working Group, ${ }^{46}$ Jyoti Nangalia, 1,3,4 \\ Anthony R. Green, ${ }^{3,4}$ Carlos Caldas, ${ }^{3,5}$ Åke Borg,,${ }^{6,7,8}$ Andrew Tutt, ${ }^{9}$ Ming Ta \\ Michael Lee, ${ }^{10,11}$ Laura J. van't Veer, ${ }^{12,13}$ Benita K.T. Tan, ${ }^{14}$ Samuel Aparicio, ${ }^{15}$ \\ Paul N. Span, ${ }^{16}$ John W.M. Martens, ${ }^{17}$ Stian Knappskog, ${ }^{18,19}$ Anne Vincent-Salomon, 20 \\ Anne-Lise Børresen-Dale, ${ }^{21,22}$ Jórunn Erla Eyfjörd, ${ }^{23}$ Ola Myklebost, ${ }^{24}$ Adrienne \\ M. Flanagan, ${ }^{25,26}$ Christopher Foster, ${ }^{27}$ David E. Neal, ${ }^{28,29}{ }^{2}$ Colin Cooper, ${ }^{30,31}$ \\ Rosalind Eeles, ${ }^{32,33}$ G. Steven Bova, ${ }^{34}$ Sunil R. Lakhani, ${ }^{35,36,37}$ Christine Desmedt, $^{38}$ \\ Gilles Thomas, ${ }^{39,44}$ Andrea L. Richardson, ${ }^{40,41}$ Colin A. Purdie, ${ }^{42}$ Alastair \\ M. Thompson, ${ }^{43}$ Ultan McDermott, ${ }^{1}$ Fengtang Yang, ${ }^{2}$ Serena Nik-Zainal, ${ }^{1}$ Peter \\ J. Campbell, ${ }^{1}$ and Michael R. Stratton ${ }^{1}$ \\ ${ }^{1-43}$ [Author affiliations appear at end of paper.]
}

\begin{abstract}
Mitochondrial genomes are separated from the nuclear genome for most of the cell cycle by the nuclear double membrane, intervening cytoplasm, and the mitochondrial double membrane. Despite these physical barriers, we show that somatically acquired mitochondrial-nuclear genome fusion sequences are present in cancer cells. Most occur in conjunction with intranuclear genomic rearrangements, and the features of the fusion fragments indicate that nonhomologous end joining and/or replication-dependent DNA double-strand break repair are the dominant mechanisms involved. Remarkably, mitochondrial-nuclear genome fusions occur at a similar rate per base pair of DNA as interchromosomal nuclear rearrangements, indicating the presence of a high frequency of contact between mitochondrial and nuclear DNA in some somatic cells. Transmission of mitochondrial DNA to the nuclear genome occurs in neoplastically transformed cells, but we do not exclude the possibility that some mitochondrial-nuclear DNA fusions observed in cancer occurred years earlier in normal somatic cells.
\end{abstract}

[Supplemental material is available for this article.]

Somatically acquired structural rearrangements are common features of the nuclear genomes of cancer cells. These may range from simple chromosomal rearrangements (Campbell et al. 2008) to more complex, compound patterns, such as chromothripsis (Stephens et al. 2011) and chromoplexy (Baca et al. 2013), or mobilization of transposable elements (Lee et al. 2012; Tubio et al. 2014). Intrachromosomal rearrangements are generally more common than interchromosomal rearrangements, indicating a higher likelihood of joining a double-strand break in a chromosome to another break in the same chromosome despite

\footnotetext{
${ }^{44}$ Deceased.

45 These authors contributed equally to this work.

${ }^{46} \mathrm{~A}$ full list of members is provided in the Supplemental Material. Corresponding author: mrs@sanger.ac.uk

Article published online before print. Article, supplemental material, and publication date are at http://www.genome.org/cgi/doi/10.1101/gr.190470.115. Freely available online through the Genome Research Open Access option.
}

the availability of a much larger quantity of nuclear DNA from other chromosomes (Stephens et al. 2009).

In addition to the nuclear genome, human cells have a few hundred to a few thousand mitochondria, each carrying one or a few copies of the 16,569-bp-long circular mtDNA (Smeitink et al. 2001; Friedman and Nunnari 2014; Ju et al. 2014). During endosymbiotic co-evolution, most of the genetic information present in the ancestral mitochondrion has transferred to the nuclear genome (Gray et al. 1999; Adams and Palmer 2003; Timmis et al. 2004). An apparent burst of mtDNA transfer occurred during primate evolution 54 million years ago (Gherman et al. 2007) and occasional, probably more recent, transfer in humans has been observed in the germline (Turner et al. 2003; Goldin et al. 2004; Chen et al. 2005; Millar et al. 2010; Dayama et al. 2014).

(C) $2015 \mathrm{Ju}$ et al. This article, published in Genome Research, is available under a Creative Commons License (Attribution 4.0 International), as described at http://creativecommons.org/licenses/by/4.0/. 
Although mtDNA nuclear transfer in a HeLa cell line derivative, and thus occurring in vitro, has been reported (Shay et al. 1991), de novo nuclear transfer of mtDNA in animal somatic tissues has not previously been comprehensively studied to our knowledge. To investigate the possibility of somatic mitochondrial-nuclear DNA fusion, we analyzed next-generation paired-end DNA whole-genome sequencing data from 559 primary cancers, 28 cancer cell lines (referred as 587 cancer whole genome below) and normal DNAs from the same individuals (Supplemental Table 1).

\section{Results}

\section{Discovery of somatic mtDNA transfers to cancer nuclear genomes}

From the 587 pairs of cancer and normal whole-genome sequencing data, we searched for cancer-specific clusters of discordant paired-end sequence reads in which one member of the read-pair mapped to the nuclear genome and the other to the mitochondrial genome, and then characterized the nuclearmitochondrial genome junctions to nucleotide resolution using individual sequence reads that bridged the junction (Fig. 1A). In 12 samples (overall positive rate $2.0 \%, 12$ out of 587 samples), we observed 25 cancer-specific mitochondrial-nuclear DNA junctions (Table 1; Supplemental Figs. 1-6). Given that there are two junctions for a single integration event, we conclude that there are most likely 16 independent mtDNA insertions (Table 1). In addition to somatic transfers, we observed several novel rare germline (inherited) events that were shared between cancer and paired normal samples (Supplemental Table 2; Supplemental Material).

Breast cancer PD11372a showed a somatically acquired integration of almost the entire human mtDNA sequence $(16,556 \mathrm{bp})$ into a highly amplified 2.75 -Mb-long region of Chromosome 10q22.3. The integration event was strongly supported by both discordant and split read clusters (Fig. 1B-D) and was confirmed by short- and long-range PCR across the nuclear-mitochondrial genome junctions (Supplemental Figs. 7, 8; Supplemental Table 3). It was not found in normal tissue (blood) from the same individual or from all the other cases and did not match any known inherited nuclear mtDNA-like sequences (known as numts) (Gherman et al. 2007; Hazkani-Covo et al. 2010). Consistent with its somatic origin, the mtDNA fused to the nuclear genome harbored sequence polymorphisms identical to those present in the mitochondria of this individual $(14,905$ G>A; 15,028 C>A; 15,043 G>A; 15,326 A> G; 15,452 C>A, and $15,607 \mathrm{~A}>\mathrm{G}$ ). Fluorescence in situ hybridization (FISH) experiments performed on formalin-fixed paraffin embedded tissue confirmed that the fused DNA segment exists in the nuclei of cancer cells (Fig. 1E).

In total, we found 10 primary cancers $(1.8 \%, 10 / 559)$ and two cancer cell lines $(7.1 \%, 2 / 28)$ with somatic mtDNA integrations into their nuclear genomes (Table 1; Supplemental Figs. 1-6). Of the 12 cancers, two (primary cancer PD13296a and cancer cell line NCI-H2087) had more than one mitochondrial-nuclear DNA translocation event. All integrations were supported by both discordant and split reads and further confirmed by PCR across the nuclear-mitochondrial genome junctions (Supplemental Fig. 7; Supplemental Table 3). All inherited mtDNA substitution polymorphisms near these breakpoints were detected (Table 1). To further visualize the transfer events, we performed high-resolution FISH on stretched DNA fibers (fiber FISH) from the melanoma cell line, CP66-MEL (Fig. 2A).
Somatic nuclear integration of mtDNA is frequently combined with other rearrangements of the nuclear genome

The rate of somatic nuclear transfer of mtDNA may vary according to tumor type. Triple-negative breast cancer showed a fivefold higher frequency compared to estrogen-receptor (ER) positive breast cancers $(6.2 \%$ and $1.2 \%$, respectively; Fisher's exact test $P$ $=0.002$ ). Triple-negative breast cancer genomes carry a higher number of chromosomal rearrangements than ER-positive breast (average 254 and 94, respectively, in our data set). As a result, there was a suggestive positive correlation between the number of chromosomal rearrangements and mtDNA transfers (Mann-Whitney $U$ test, one-sided $P=0.05$ ) (Fig. 2B).

The length of mtDNA fragments transferred ranged from 148 bp to entire mitochondrial genomes $(16.5 \mathrm{~kb})$ (Table 1$)$. Interestingly, breakpoints in mtDNA were enriched near the mitochondrial genome heavy strand origin of replication $\left(\chi^{2}\right.$ test, $\left.P=0.0005\right)$ (Fig. 2C). This suggests that the generation of mtDNA segments to be integrated into the nuclear genome is not random and may occur in a mtDNA replication-dependent manner (Lenglez et al. 2010).

Of the 25 mitochondrial-nuclear DNA junctions, at least 17 $(68.0 \%)$ were clearly associated with other nuclear chromosomal rearrangements (e.g., inversions, translocations, and large deletions) in the vicinity (Table 1; Supplemental Figs. 1-6). For example, with respect to PD11372a described earlier, genomic fragments from Chromosomes 10, 11, and mtDNA generated complex derivative chromosomes (Fig. 3A). In PD6047a, an mtDNA fragment was involved in chains of complex genomic translocations involving Chromosomes 6, 7, 11, 22, and X (Fig. 3B). In PD10014a, a local inversion was combined with the mtDNA integration event (Fig. 3C), and in PD4252a, a 16.5-kb mtDNA integration was found in a position on the $\mathrm{X}$ Chromosome from which $\sim 20 \mathrm{~kb}$ of nuclear DNA had been somatically deleted (Fig. 3D). Thus, mtDNA is often integrated into nuclear genomes in the vicinity of, or as part of, complex rearrangements. Although germline numts tend to occur near transposable elements such as LINEs and SINEs (Mishmar et al. 2004), we do not observe this association for somatic events $\left(\chi^{2}\right.$ test, two-sided $P=0.33$ ) (Supplemental Table 4).

\section{The mechanism and timing of somatic nuclear transfer of mtDNA}

There was overlapping sequence microhomology (from 1 to $4 \mathrm{bp}$ ) in 20/25 breakpoints (80\%) (Fig. 4A,B; Table 1; Supplemental Figs. $1-6)$, substantially more than expected by chance $\left(\chi^{2}\right.$ test, $P=5 \times$ $\left.10^{-26}\right)$. Thus, DNA sequence microhomology plays an important role in mitochondrial-nuclear DNA integration events, although blunt-end DNA repair was also observed. In two breakpoints, we also found nontemplated short-nucleotide insertions (1 and $4 \mathrm{bp}$ long) (Fig. 4A; Table 1). Overall, these features are characteristic of DNA double-strand break repair by nonhomologous end joining (NHEJ) (Hastings et al. 2009). However, they do not rule out replication-based mechanisms switching template between nuclear and mtDNA, such as microhomology-mediated break-induced replication (MMBIR) (Liu et al. 2011).

We investigated the timing of somatic mtDNA integration into the nuclear genome by assessing cases in which a metastatic sample had been sequenced in addition to the primary tumor. One such case (PD4252a) showed the mitochondrial-nuclear integration event in the primary but not in the metastasis (Fig. 4C), indicating that mtDNA transfer to the nucleus can occur after 
A

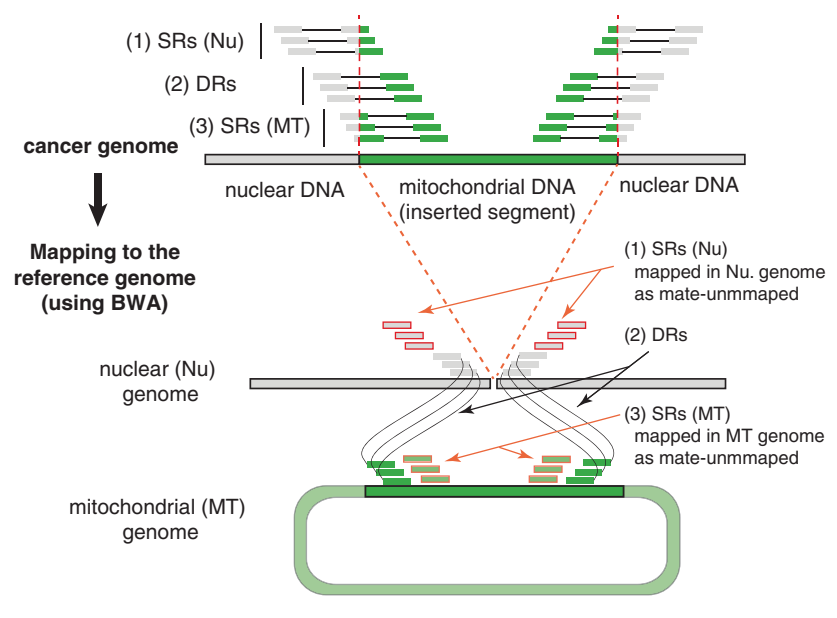

B

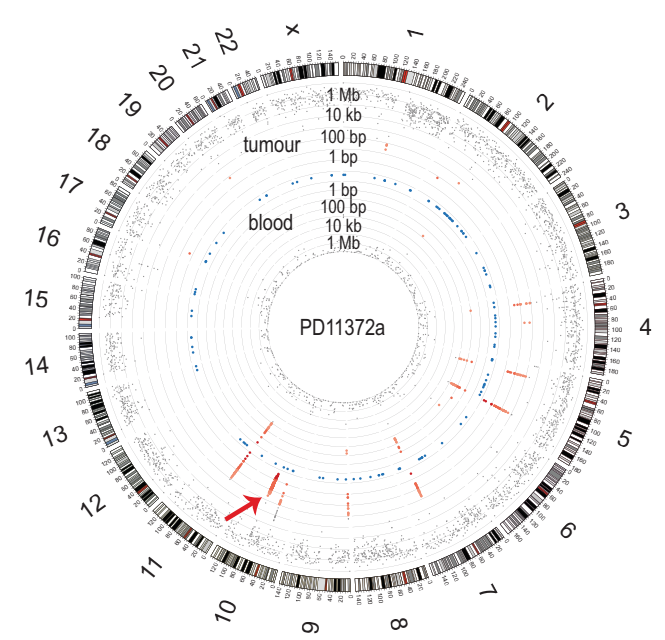

C

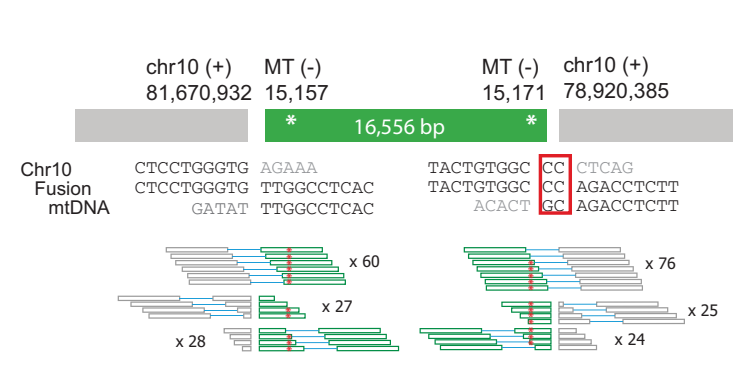

D

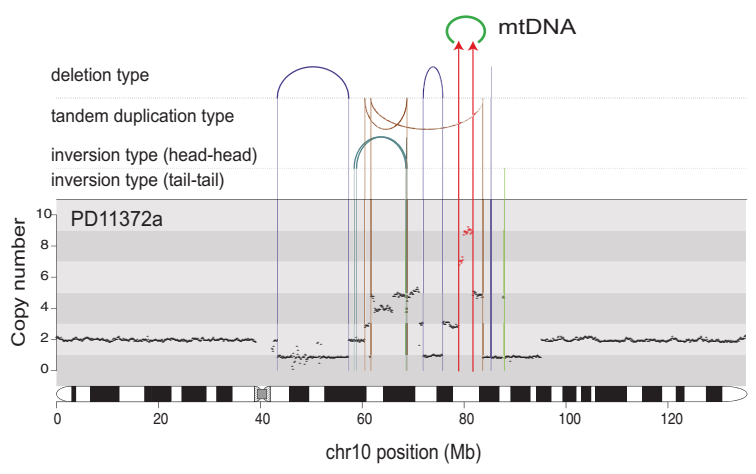

E
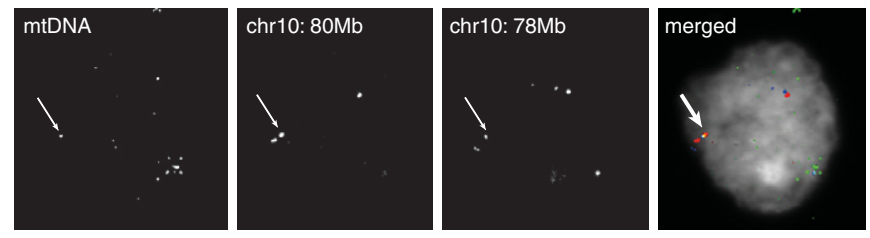

Figure 1. Discovery of somatic nuclear mtDNA transfer from PD11372a. (A) The strategy for detection of nuclear mtDNA transfer events. See Methods for a detailed description. (SRs) Split-reads, (DRs) discordant reads, (Nu) nucleus, (MT) mitochondria. (B) Graphical representation of discordant read clusters in PD11372a and its paired-normal tissue (PD11372b). The red arrow indicates tumor-specific discordant-read clusters in Chr 10. Chromosome ideograms are shown in the outer layer. The distance between each discordant read and one prior to it (the inter-read distance) is plotted on the vertical axis on a log-scale in the middle (tumor) and inner layer (blood). Blue dots shown in the middle layer represent known numts. (C) mtDNA integration in PD11372a. Breakpoint sequences are shown. Red rectangle highlights microhomology. Numbers of discordant split reads are presented. Inherited mtDNA substitution polymorphisms are shown by red asterisks. $(D)$ Rearrangement architectures of Chromosome 10 of PD11372a. DNA copy numbers are shown by black dots. The copy number for 2.75-Mb-long region fused with mtDNA is colored in red. Reads supporting rearrangements (large deletions, tandem duplications, tail-tail and head-head inversions) are shown by arcs and vertical lines. Chr 10-mtDNA fusions are shown with red arrows. (E) Nuclear FISH confirms the mitochondrial-nuclear DNA fusion in the nucleus. (Red) Chr 10 (80 Mb), (blue) Chr 10 (78 Mb), and (green) mtDNA.

neoplastic transformation and during the course of subclonal evolution of the cancer. The other (PD6728b) showed it in both the primary and metastasis (Fig. 4C), suggesting that this event occurred in the common ancestral cancer clone or in normal somatic cells prior to neoplastic change.
Nuclear transfer of mtDNA is unexpectedly frequent in human somatic cells

To obtain a perspective on the frequency of mitochondrial-nuclear DNA translocation, we compared its rate to that of intranuclear

\section{Genome Research}

www.genome.org 


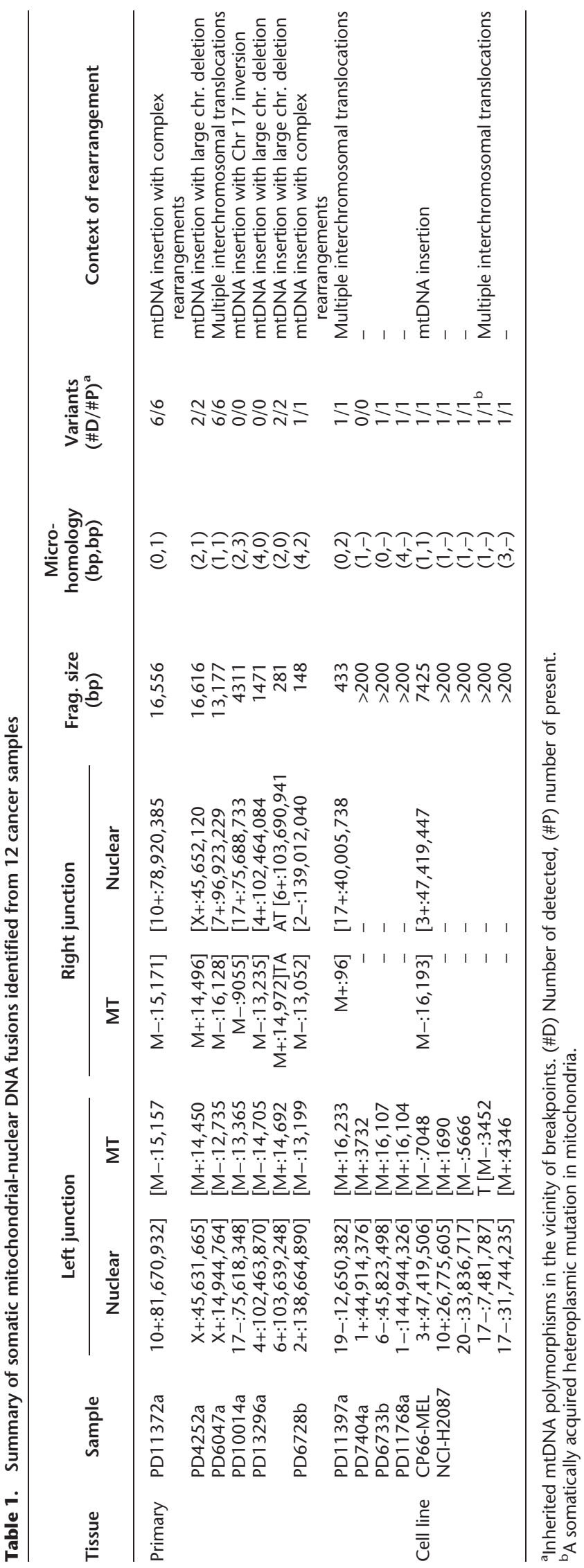


A

CP66-MEL (melanoma cell-line)

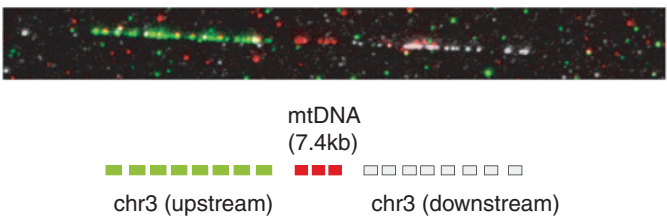

B

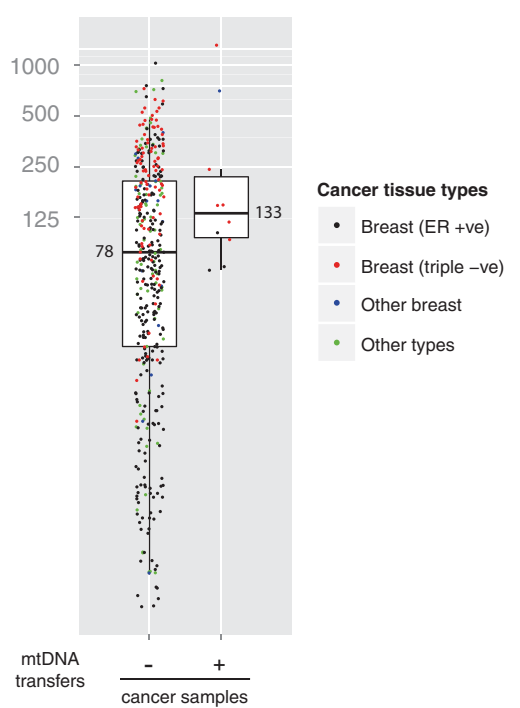

C

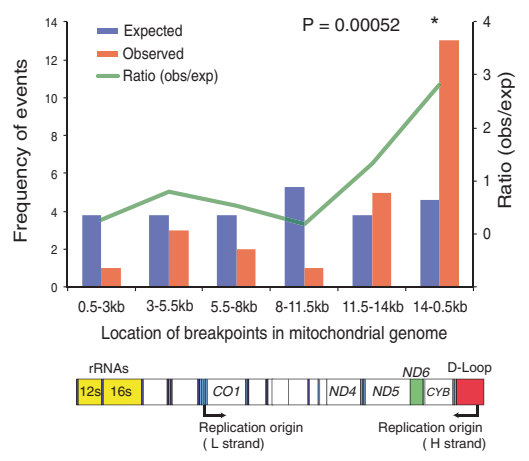

Figure 2. Features of somatic mtDNA nuclear transfer in 12 cancer samples. (A) Fiber FISH visualizes the mitochondrial-nuclear DNA fusion from the CP66-MEL cell line. (B) Positive correlation between mtDNA transfer and numbers of nuclear chromosomal rearrangements (large deletion, tandem duplication, inversion, and translocation) in cancer genomes. Median values are shown. (C) mtDNA breakpoints are enriched in the $14 \mathrm{~kb}$ - to 500-bp region of the MT genome. (Top) Blue and red bars represent the expected and observed numbers of breakpoints in each interval of MT genome, respectively. Green line shows ratio between observed and expected numbers. A $\chi^{2}$ test was applied to test enrichment. (Bottom) Schematic structural features of the MT genome corresponding to the intervals are shown.

interchromosomal translocation, taking into account the sizes and copy numbers of the mitochondrial and nuclear genomes. Our sequencing data suggest that each cancer cell carries $\sim 500$ copies of circular mtDNA (median value 495) (Fig. 5A), amounting in aggregate to $\sim 8$ million base pairs (bp) of mtDNA (500 copies $\times 16.5 \mathrm{~kb}$ ) enclosed by the mitochondrial double membrane in the cytoplasm of each cancer cell. The average frequency in the cancers analyzed of mitochondrial-nuclear DNA fusion was $5.1 \times 10^{-3}$ junctions per million bp of mtDNA, only half the average rate of intranuclear interchromosomal translocation $\left(1.2 \times 10^{-2}\right.$ junctions per million bp) and similar to that of Chromosomes 2, 4, and 13 (Fig. 5B). Given the multiple physical barriers to contact between the two genomes, the results indicate remarkably high rates of mtDNA escape, contact, and/or integration with nuclear DNA in human cancer cells. These appear to be considerably higher than in the germline across human evolution but comparable to those observed in Saccharomyces cerevisiae (Thorsness and Fox 1990) and for chloroplast DNA migration into the nucleus in tobacco plants (Methods; Supplemental Material; Huang et al. 2003).

\section{Discussion}

Despite multiple physical barriers, there are plausible mechanisms by which mtDNA and nuclear DNA could come into contact (Fig. 5C). Free mtDNA can be released into the cytoplasm from degrading mitochondria or after mitophagy (Zhang et al. 2008; Eiyama et al. 2013; Higgins and Coughlan 2014). Degradation of mitochondria may be accelerated in cancer cells due to hypoxia and increased energy demands (Zhang et al. 2008; Eiyama et al. 2013; Higgins and Coughlan 2014). Even without a bespoke molecular process for transportation, mtDNA could then, in principle, migrate to the nucleus during mitotic metaphase or anaphase when the nuclear membrane has broken down. When these events are coupled with concurrent double-strand breaks (DSBs) and/or replication fork stalling of nuclear chromosomal DNA, mtDNA could be picked up and integrated into the nuclear genome as part of the process of rejoining DSBs (NHEJ) (Hastings et al. 2009) or used as an alternative DNA template in replication (MMBIR) (Liu et al. 2011). Micronuclei in cancer cells, which can be generated by errors in segregation of mitotic nuclear chromosomes, may contribute to the events. Chromosomes in micronuclei frequently undergo defective and delayed DNA replication, resulting in extensive fragmentation with subsequent jumbled rejoining compared to their original order and orientation (Crasta et al. 2012; Forment et al. 2012). Thus, mtDNA fragments incorporated into micronuclei could end up fused to shattered nuclear chromosomes. It is worthy of note that mtDNA escaping to the nucleus can be actively used for DNA repair in Saccharomyces cerevisiae (Ricchetti et al. 1999; Yu and Gabriel 1999), particularly when error-free DSB DNA repair is not possible. Whether this applies in mammalian cells is unknown.

Some of the somatic nuclear mtDNA integrations we identified are directly adjacent to nuclear genes. For example, nuclearmtDNA fusion in PD11372a occurred in the fifth intron of the KCNMA1 gene, a potassium channel frequently amplified in prostate and breast cancers (Oeggerli et al. 2012). However, we do not find obvious enrichment of the nuclear-mtDNA fusion breakpoints near human nuclear genes. RNA-seq from the NCI-H2087 cell-line indicates that mtDNA fragments in the nucleus of the cell line are not expressed as parts of mitochondrial-nuclear fusion transcripts. Thus, the majority of the nuclear mtDNA translocation events are likely to be passenger events, similar to mutations of all other types in most cancer genomes. However, we do not exclude the possibility that some of these events may have functional consequences in human cancer by generating fusion mRNA transcripts (Shay et al. 1991) and/or truncating cancer genes by mtDNA insertion within exons.

\section{Genome Research}

www.genome.org 
A

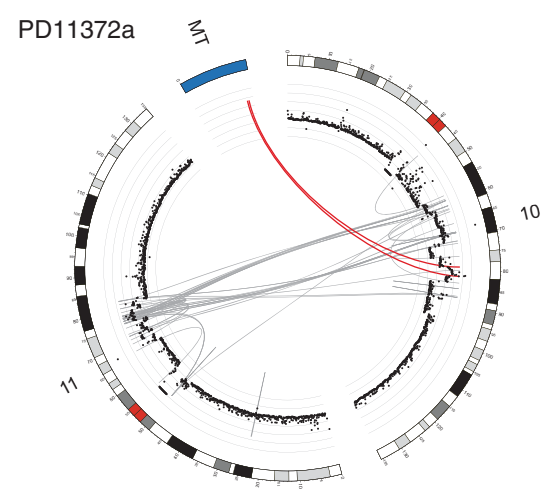

B
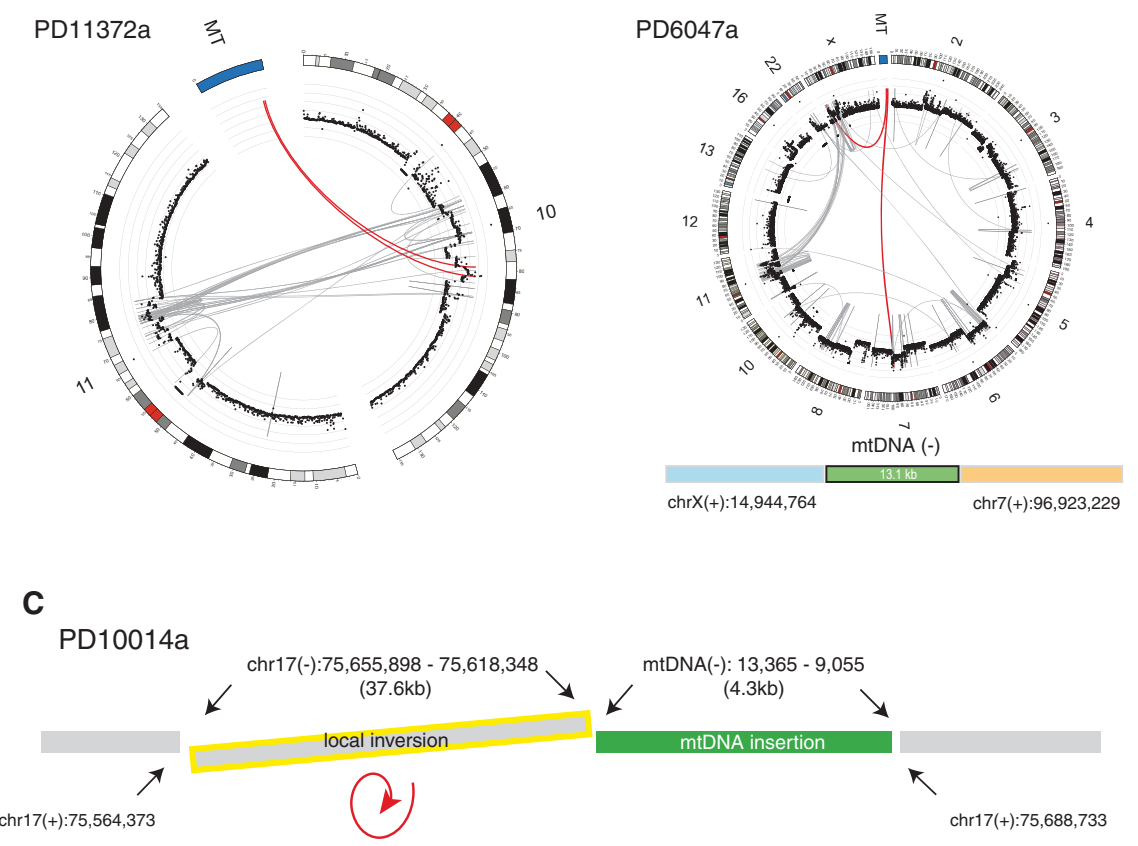

D

\section{PD4252a}

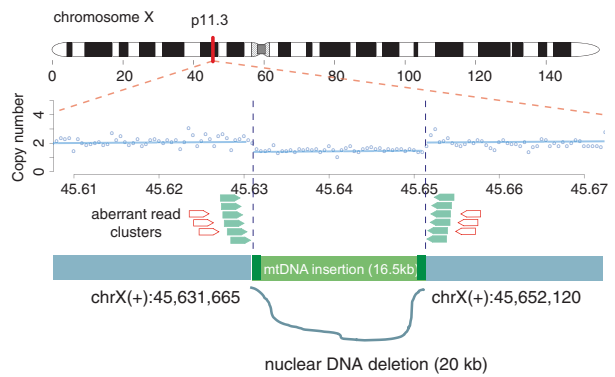

Figure 3. Concurrence of somatic mtDNA nuclear transfers with other structural variations. The complex web of rearrangements in the vicinity of mitochondrial-nuclear DNA fusions from four examples. $(A)$ In PD11372a, mtDNA integration with complex rearrangements between $\mathrm{Chr} 10$ and 11. (B) In PD6047a, mtDNA integration with complex rearrangements among Chr 6, 7, 11, 22, and X. $(A, B)$ DNA copy numbers are shown by black dots with a log scale. Red lines represent translocations involving mtDNA. (C) In PD10014a, mtDNA integration combined with a local inversion (yellow). (D) In PD4252a, mtDNA integration with a local deletion. DNA copy numbers are shown with blue dots and lines. Aberrant read clusters (discordant and split reads) are shown by green and red arrows, respectively.

This study has shown that fusion of mtDNA to nuclear DNA occurs in human somatic cells at a rate similar to that of translocation between nuclear chromosomes. Physical migration of mtDNA into the nucleus may be much more frequent in stem cells than ones in a terminally differentiated stage (Schneider et al. 2014). Further studies will need to address the mechanisms by which the apparent physical barriers to contact between mitochondrial and nuclear DNA are so effectively overcome.

\section{Methods}

\section{Samples and sequencing data}

We analyzed 559 primary tumors and 28 cancer cell-lines in this study. Paired-normal samples for all the cancers were also included in this study in parallel. Whole-genome sequences used in this study were generated by Illumina platforms (either Genome Analyzer or HiSeq 2000). Cancer genomes were sequenced to at least $25 \times \mathrm{cov}$ erage. With respect to TCGA data, we downloaded aligned BAM files through UCSC CGHub (http://cghub.ucsc.edu). Sequencing reads were aligned on the human reference genome build 37 (GRCh37) and human reference mtDNA sequence (revised Cambridge reference sequence, rCRS) (Andrews et al. 1999), mainly by the BWA alignment tool (Li and Durbin 2009). SAMtools (Li et al. 2009) was used for manipulating sequence reads.

\section{Calling mitochondrial-nuclear DNA fusion events}

We employed a pipeline for identification of putative mtDNA translocation to chromosomal DNA (Fig. 1A). From paired-end whole-genome sequencing data of tumors, we extracted discordant reads (DRs), where one end aligned uniquely to mtDNA and the other end to nuclear DNA. In all cases, both ends must have a mapping quality greater than zero. Those discordant reads are clustered together using the following criteria: reads sharing (1) close alignment positions ( $<500$ nucleotides) for both ends on nuclear and mtDNA, and (2) the same orientations. In order to remove false positives, we removed clusters supported by less than five discordant reads.

In order to remove potential germline calls, several filters are applied to the tumor candidate cluster. The clusters from tumor cells were removed if they overlap with clusters identified from matched and/or unmatched normal tissues by more tolerable criteria (supported by more than one discordant read) from (1) its paired-normal tissue, and (2) from the other 586 unmatched normals. Filtered clusters were further refined with known germline human numts, a combined set from the human reference genome (hg19) detected by BLAT (Kent 2002) $(n=123)$ and from Simone et al. (2011) $(n=766)$. Finally, 25 clusters were selected as somatic candidates.

Nucleotide-resolution breakpoints for the translocation junctions To obtain nucleotide-resolution breakpoints, we searched for splitreads (SRs) with one of the ends spanning the junction of the translocation. We extracted "orphan" or "mate-unmapped" reads (one end of a read is unmapped by the BWA aligner) in the vicinity $(<1000$ bp) of discordant-read clusters on nuclear and mitochondrial genome sequences. Sequences from the unmapped end are then re-aligned by BLAT (Kent 2002), which enables split-read mapping. 
A

PD13296a (2 mtDNA nuclear integration events)

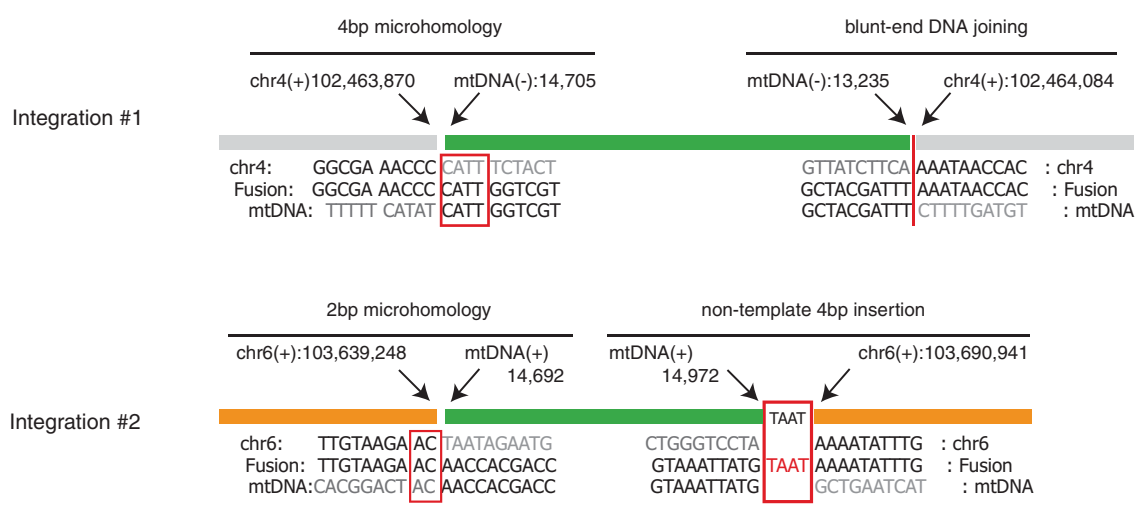

B

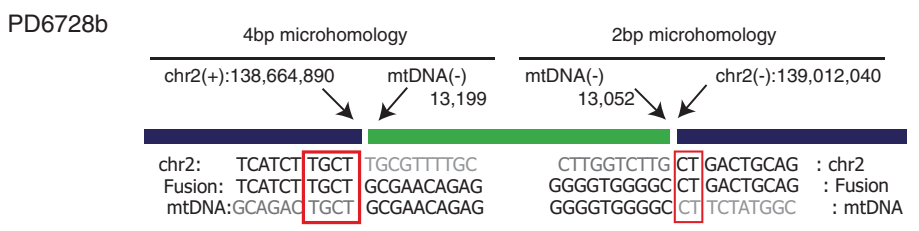

C
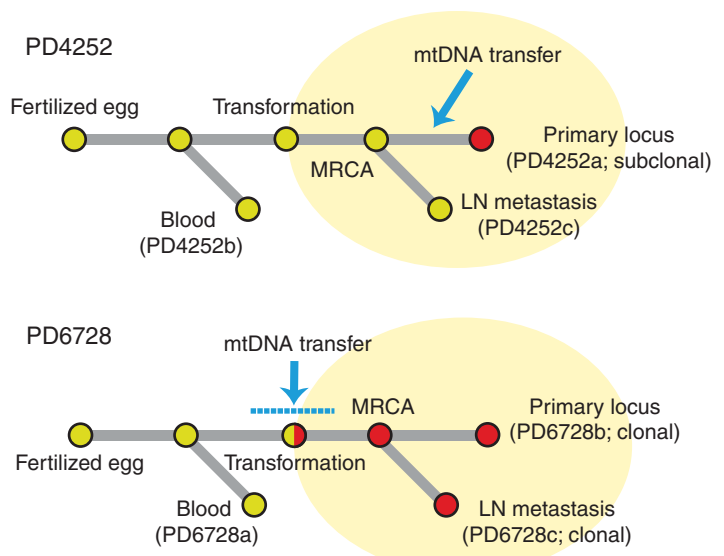

Figure 4. Nucleotide-resolution breakpoint sequences and the timing of somatic mtDNA nuclear integration. $(A)$ Breakpoint sequences of nuclear-mtDNA fusions in PD13296a. Red rectangles highlight sequence microhomology and nontemplate nucleotides insertion. (B) Breakpoint sequences of nuclearmtDNA fusions in PD6728b. Red rectangles highlight sequence microhomology. (C) Phylogenetic trees showing the timing of somatic mtDNA nuclear transfers in PD4252 and PD6728 samples. (MRCA) Most recent common ancestor cell.

\section{Validation by PCR}

A PCR validation assay of the somatic mtDNA transfer was performed using genomic DNA from both cancer and paired-normal tissues. Primers were designed to amplify all the breakpoints (Supplemental Table 3). The short-fragment PCR reactions were performed as previously described (Tubio et al. 2014). With respect to long-range PCR, elongation time was increased 1 min per $1 \mathrm{~kb}$.

\section{Generation of FISH probes}

Human bacterial artificial chromosomes (BAC) and fosmid clones used in this study were obtained from the clone archive team of the
Wellcome Trust Sanger Institute. Plasmid DNA was prepared using the PhasePrep BAC DNA kit (Sigma-Aldrich). Human mtDNA was isolated from lymphoblastoid cells using a Mitochondrial DNA Isolation kit (Abcam).

Probes for use in FISH were made as described before (Gribble et al. 2013). Purified mtDNA and plasmid DNA were first amplified using a GenomePlex Whole Genome Amplification (WGA) kit (Sigma-Aldrich) following the manufacturer's protocols, then labeled using a WGA reamplification kit (SigmaAldrich) with a custom-made dNTP mix. Probes for interphase FISH were labeled directly with Aminoallyl-dUTPs - ATTO488, -Cy3, -Texas Red, and -Cy5 (Jena Bioscience); probes for fiber-FISH were labeled with Biotin-16-dUTP, Digoxigenin11-dUTP (Roche), and DNP-11-dUTP (PerkinElmer).

\section{Validation by fiber-FISH with single- molecule DNA fibers generated by molecular combing}

Single-molecule DNA fibers from the cancer cell line, CP66-MEL, were prepared by molecular combing (Michalet et al. 1997) following the manufacturer's instructions (Genomic Vision). Briefly, the cells were embedded in a low-meltpoint agarose plug ( 1 million cells per plug), followed by proteinase $\mathrm{K}$ digestion, washing in $1 \times \mathrm{TE}(10 \mathrm{mM}$ Tris, 1 mM EDTA, pH 8.0) and beta-agarose digestion steps. The DNA fibers were mechanically stretched onto saline-coated coverslips using a Molecular Combing System (Genomic Vision).

For fiber-FISH, $500 \mathrm{ng}$ of labeled DNA from each probe and $4 \mu \mathrm{g}$ of human Cot-1 DNA (Invitrogen) were precipitated using ethanol, then resuspended in a mix (1:1) of hybridization buffer (containing $2 \times$ SSC, 10\% sarkosyl, $2 \mathrm{M}$ $\mathrm{NaCl}, 10 \%$ SDS, and blocking aid [Invitrogen]) and deionized formamide (final concentration 50\%). Coverslips coated with combed DNA fibers were dehydrated through a 70\%, 90\%, and $100 \%$ ethanol series and aged at $65^{\circ} \mathrm{C}$ for $30 \mathrm{sec}$, followed by denaturation in an alkaline denature solution $(0.5 \mathrm{M} \mathrm{NaOH}, 1.5$ $\mathrm{M} \mathrm{NaCl}$ ) for 1-3 min, three washes with $1 \times \mathrm{PBS}$ (Invitrogen), and dehydration through a $70 \%, 90 \%$, and $100 \%$ ethanol series. The probe mix was denatured at $65^{\circ} \mathrm{C}$ for $10 \mathrm{~min}$ before being applied onto the coverslips, and the hybridization was carried out in a $37^{\circ}$ $\mathrm{C}$ incubator overnight. The post-hybridization washes consisted of two rounds of washes in $50 \%$ formamide $/ 2 \times$ SSC $(v / v)$, followed by two additional washes in $2 \times$ SSC. All post-hybridization washes were done at $25^{\circ} \mathrm{C}, 5 \mathrm{~min}$ each time. Digoxigenin-11-dUTP (Roche) labeled probes were detected using a 1:100 dilution of monoclonal

\section{Genome Research}

www.genome.org 
A

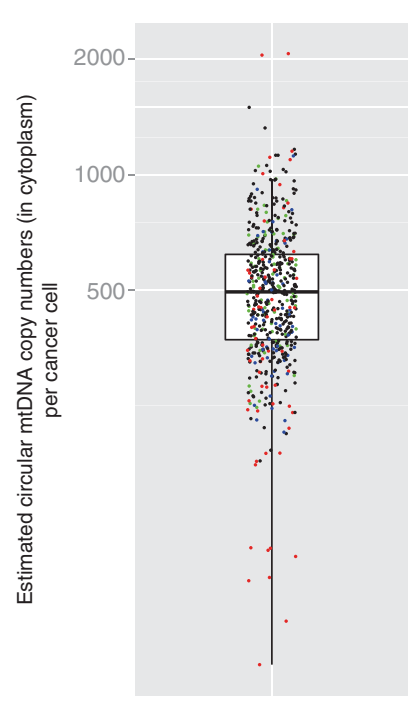

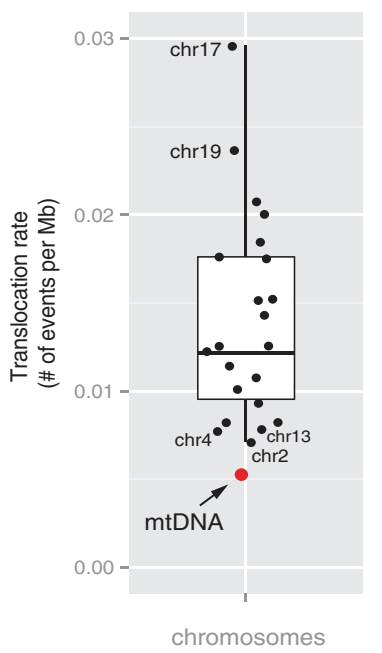

C
B

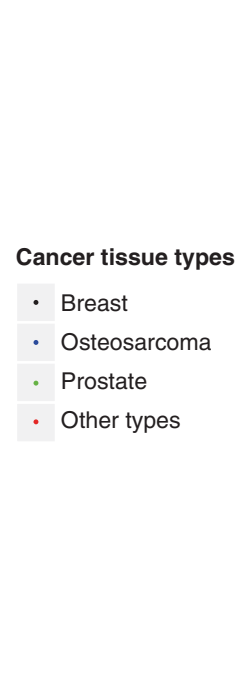

chromosomes

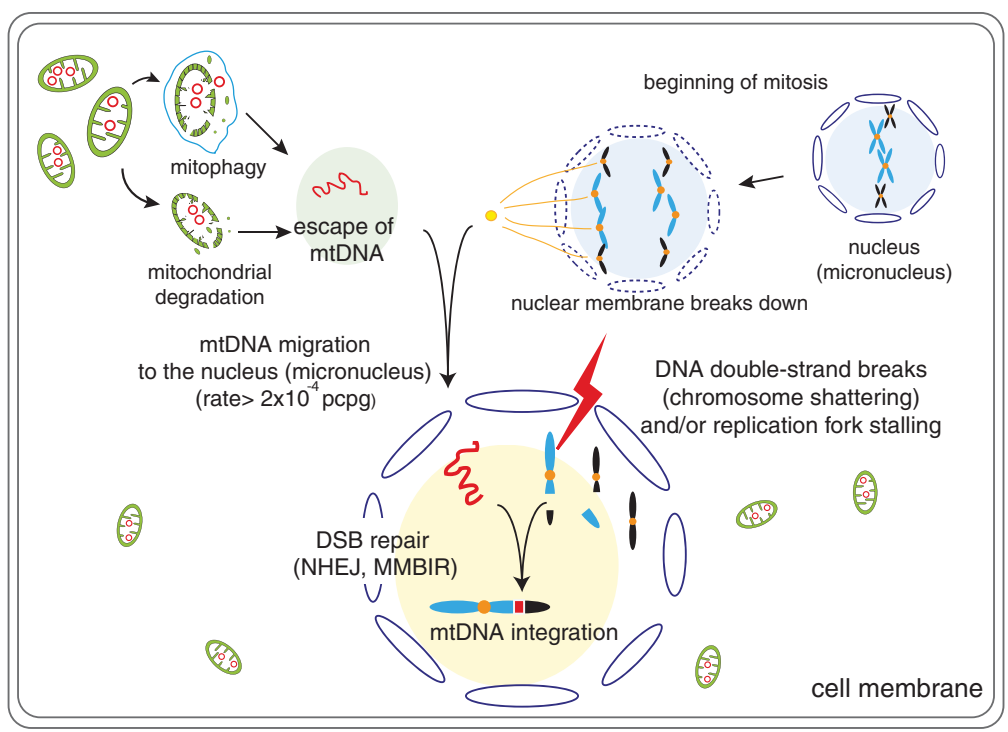

Figure 5. Frequency and potential mechanisms of somatic mtDNA nuclear transfer in human cancer. (A) Estimated circular mtDNA copy numbers (in the cytoplasm) per cancer cell from 587 cancer tissues sequenced. The ratio of read depths between autosomes and mtDNA was used (see Methods). (B) Similar frequency of somatic nuclear mtDNA integrations compared to the frequency between autosomes (chromosomal translocation). (C) A model of somatic mtDNA transfer to the nuclear genomes.

mouse anti-dig antibody (Sigma-Aldrich) and a 1:100 of Texas Red$\mathrm{X}$-conjugated goat anti-mouse IgG (Molecular Probes/Invitrogen); DNP-11-dUTP (PerkinElmer) labeled probes were detected using a 1:100 dilution of Alexa 488-conjugated rabbit anti-DNP IgG and 1:100 Alexa 488-conjugated donkey anti-rabbit IgG (Molecular Probes/Invitrogen); biotin-16-dUTP (Roche) labeled probes were detected with one layer 1:100 of Cy3-avidin (Sigma-Aldrich). After detection, slides were mounted with SlowFade Gold mounting solution containing 4',6-diamidino-2-phenylindole (Molecular Probes/Invitrogen). Images were visualized on a Zeiss AxioImager D1 microscope. Digital image capture and processing were carried out using the SmartCapture software (Digital Scientific UK).

\section{Nuclear interphase FISH}

Nuclei extraction from paraffin-embedded tissue of patient PD11372a and interphase-FISH followed Paternoster et al. (2002), with the exception that $60-\mu \mathrm{m}$ thick sections were used in our study. The post-hybridization washes consisted of two rounds of washes in $50 \%$ formamide/ $2 \times$ SSC (v/v), followed by two additional washes in $2 \times$ SSC. Slides were mounted with SlowFade Gold mounting solution containing 4',6-diamidino-2-phenylindole (Molecular Probes/ Invitrogen). Images were captured and processed as described above.

\section{Correlation between somatic mtDNA integration site and transposable elements}

We performed a study similar to the previous report (Mishmar et al. 2004). We calculated the distance between each mtDNA-insertion site (breakpoint) and its nearest transposable elements (either of SINE, LINE, LTR, simple repeat, or DNA transposon by RepeatMasker, downloaded from the UCSC Genome Browser, June 6, 2013). Then, each mtDNA-insertion site was categorized into one of four groups: (A) breakpoint within a transposable element; (B) breakpoint within $15 \mathrm{bp}$ from a transposable element; (C) within 15-150 bp; and (D), $>150 \mathrm{bp}$. In order to understand the positional enrichment of breakpoints from transposable elements, we randomly generated in silico breakpoint positions 40 times as many (total $n=1000$ ) as we observed from each chromosome in the real data set. In silico breakpoints located within gaps of the human reference genome were removed and replaced by newly generated insertions. For these in silico-generated breakpoints, the distances from the nearest transposable elements were calculated and then categorized into one of the four groups (A, B,C, and D). Finally, the difference in the frequency of breakpoints in each group between the observed and in silico-generated data set was compared using a $\chi^{2}$ test.

\section{Assessment of mtDNA copy numbers}

To understand mtDNA copy numbers in a cancer cell, we compared average read depth of coverage between 22 autosomes and mtDNA. With respect to the tumor sequences by whole-genome sequencing, average haploid autosomal coverage ( $\left.\mathrm{RD}_{\text {autosome }}\right)$ was obtained from the read depth of 2.685-Gb-long autosomal regions (excluding chromosomal gaps). Likewise, average mtDNA coverage $\left(\mathrm{RD}_{\mathrm{mtDNA}}\right)$ was obtained from the read depth of the 
16.5-kb mitochondrial genome. Finally, mtDNA copy number in a diploid cell $\left(C_{\mathrm{mt}}\right)$ is calculated as shown below:

$$
C_{\mathrm{mt}}=2 \times \frac{\mathrm{RD}_{\mathrm{mtDNA}}}{\mathrm{RD}_{\text {autosome }}} .
$$

\section{Assessment of translocation rate for autosomes and mitochondria}

We identified structural variations among nuclear chromosomes (large deletions, tandem duplications, inversions, and interchromosomal translocations) using the BRASS II algorithm (NikZainal et al. 2012), which identifies rearrangements by clustering discordant read pairs that point to the same junction and confirms breakpoints by local assembly of unmapped reads. The sensitivity and specificity of the BRASS II algorithm is equivalent to those values of the algorithm used for mitochondrial-nuclear DNA fusions (data not shown). We extracted interchromosomal translocations to calculate the rate of such events. The rate of each haploid autosome (Rtr,ch) is calculated as shown below:

Rtr, ch(events per megabase $)=\mathrm{Ntr}, \mathrm{ch} /(2 \times \mathrm{Lch}) / \mathrm{Nsam}$,

where Ntr,ch is the total number of somatic interchromosomal translocation junctions involving a specific chromosome, Lch is the length of the nonredundant region of the chromosome in megabases, and Nsam is the total number of samples analyzed. To obtain the unique region length (Lch), we excluded redundant (or highly repetitive) sequence lengths from the ungapped length of each chromosome. Genomic regions classified in one or more of the three criteria shown below were defined as redundant, where translocation events could not be easily detected due to ambiguous read alignment: (1) simple repeats, located by Tandem Repeats Finder (Benson 1999); (2) segmental duplications with moderate to high sequence similarity ( $\geq 95 \%$ ) (Bailey et al. 2002), or (3) repetitive sequences including up to 10 different classes of repeats (such as SINE, LINE, LTR, DNA transposons, and microsatellites), located by the RepeatMasker program (http://www.repeatmasker.org), with a low divergence level (divergence $<5 \%$ ). These nonredundant sequence regions were downloaded from the UCSC Genome Browser (http://genome.ucsc.edu).

Similarly, the rate of mitochondrial-nuclear DNA translocations (Rtr,mt) was calculated as below:

Rtr, $\mathrm{mt}($ events per megabase $)=\mathrm{Ntr}, \mathrm{mt} /\left(C_{\mathrm{mt}} \times L_{\mathrm{mtDNA}}\right) / \mathrm{Nsam}$,

where Ntr,mt is the total number of junctions of somatic mitochondrial-nuclear DNA fusions identified, $C_{\mathrm{mt}}$ is the median value of mitochondrial genome copy numbers in a diploid cancer cell calculated above (495 copies), and $L_{\mathrm{mtDNA}}$ is the length of the mitochondrial genome in megabases $(0.016569 \mathrm{Mb})$.

\section{Assessment of the rates of nuclear mtDNA fusion and mtDNA escape to the nucleus}

Fusion of mtDNA to the nuclear genome requires at least two events, each of which could influence the rate of mitochondrialnuclear DNA fusion. These include escape of mtDNA to the nucleus and integration to nuclear DNA. According to this model, the overall number of such fusion events can be calculated using the rates for these processes ( $\rho_{\text {escape }}$ and $\rho_{\text {integration, }}$ respectively):

$$
\text { Ntr }=\text { Nsam } \times \text { Ngen } \times \rho_{\text {escape }} \times \rho_{\text {integration }},
$$

where Ntr is the number of total somatic mitochondrial-nuclear DNA fusion events $(n=12)$, Nsam is the total number of can- cer tissues $(n=587)$, and Ngen is the number of average cell generation from the fertilized egg. Using a reasonable assumption that Ngen $=1000$, we obtain the rate of somatic mtDNA fusion to the nuclear genome $\left(\rho_{\text {escape }} \times \rho_{\text {integration }}\right)$ to be $2 \times 10^{-5}$ per cell per cell generation (pcpg). With one more very conservative assumption that $\rho_{\text {integration }}$ is 0.1 , we obtain $\rho_{\text {escape }}$ to be $2 \times 10^{-4} \mathrm{pcpg}$, or at least one escape event per 5000 cell generations. We hypothesize that the real $\rho_{\text {integration }}$ value is thought to be much lower than 0.1 , which results in a higher $\rho_{\text {escape }}$. For example, during the generation of knockout mice, homologous recombination allows one fixation event per 1000-10,000 microinjected DNA copies (Brinster et al. 1985). The integration rate may, however, be higher than the rate in cancer cells with defective homologous recombination-based repair and increased availability of nuclear double-strand breaks, which can be joined to by NHEJ or MMBIR.

The mtDNA fusion to the nuclear genome in the germline (the rate of numts insertion) is around $5 \times 10^{-6}$ per germ cell per individual generation in previous phylogenetic studies (HazkaniCovo et al. 2010). The rate is equivalent to $\sim 5 \times 10^{-8} \mathrm{pcpg}$, given that the number of germ cell divisions per human generation is $\sim 100$ (401 in males and 31 in females [Drost and Lee 1995]).

\section{Data access}

Sequence data for sample pairs with positive mtDNA nuclear transfer have been submitted to the European Genome-phenome Archive (EGA; https://www.ebi.ac.uk/ega/home). The study accession number is EGAS00001001234. Sample accession numbers are available in Supplemental Table 1.

\section{List of affiliations}

${ }^{1}$ Cancer Genome Project, Wellcome Trust Sanger Institute, Hinxton, Cambridge CB10 1SA, United Kingdom; ${ }^{2}$ Cytogenetics Facility, Wellcome Trust Sanger Institute, Hinxton, Cambridge CB10 1SA, United Kingdom; ${ }^{3}$ Cambridge University Hospitals NHS Foundation Trust, Cambridge CB2 OQQ, United Kingdom; ${ }^{4}$ Department of Haematology, University of Cambridge, Cambridge CB2 OXY, United Kingdom; ${ }^{5}$ Cancer Research UK (CRUK) Cambridge Institute, University of Cambridge, Cambridge CB2 ORE, United Kingdom; ${ }^{6}$ BioCare, Strategic Cancer Research Program, SE-223 81 Lund, Sweden; ${ }^{7}$ CREATE Health, Strategic Centre for Translational Cancer Research, SE-221 00 Lund, Sweden; ${ }^{8}$ Department of Oncology and Pathology, Lund University Cancer Center, SE-221 85 Lund, Sweden; ${ }^{9}$ Breakthrough Breast Cancer Research Unit, Research Oncology, King's College London, Guy's Hospital, London SE1 9RT, United Kingdom; ${ }^{10}$ Laboratory for International Alliance on Genomic Research, RIKEN Center for Integrative Medical Sciences, 230-0045 Yokohama, Japan; ${ }^{11}$ National Center for Genome Medicine, Institute of Biomedical Sciences, Academia Sinica, Taipei 115, Taiwan; ${ }^{12}$ Department of Laboratory Medicine, Helen Diller Family Comprehensive Cancer Center, University of California, San Francisco, California 94158, USA; ${ }^{13}$ Netherlands Cancer Institute, 1066 CX Amsterdam, Netherlands; ${ }^{14}$ Department of General Surgery, Singapore General Hospital, Singapore 169608; ${ }^{15}$ Department of Molecular Oncology, British Columbia Cancer Agency, Vancouver V5Z 1L3, Canada; ${ }^{16}$ Department of Radiation Oncology and Department of Laboratory Medicine, Radboud University Medical Center, 6525 HP Nijmegen, Netherlands; ${ }^{17}$ Department of Medical Oncology, Erasmus MC Cancer Institute, Erasmus

\section{Genome Research}

www.genome.org 
University Medical Center, 3015 CE Rotterdam, Netherlands; ${ }^{18}$ Section of Oncology, Department of Clinical Science, University of Bergen, N-5020 Bergen, Norway; ${ }^{19}$ Department of Oncology, Haukeland University Hospital, 5021 Bergen, Norway; ${ }^{20}$ Institut Curie, INSERM U934 and Department of Tumor Biology, 75248 Paris cédex 05, France; ${ }^{21}$ Department of Genetics, Institute for Cancer Research, Oslo University Hospital, The Norwegian Radium Hospital, Montebello, 0310 Oslo, Norway; ${ }^{22}$ The K.G. Jebsen Center for Breast Cancer Research, Institute for Clinical Medicine, Faculty of Medicine, University of Oslo, 0450 Oslo, Norway; ${ }^{23}$ Cancer Research Laboratory, University of Iceland, 101 Reykjavik, Iceland; ${ }^{24}$ Department of Tumor Biology, Institute for Cancer Research, Oslo University Hospital, The Norwegian Radium Hospital, Montebello, Nydalen 0424 Oslo, Norway; ${ }^{25}$ Royal National Orthopaedic Hospital, Middlesex HA7 4LP, United Kingdom; ${ }^{26} \mathrm{UCL}$ Cancer Institute, University College London, London WC1E 6DD, United Kingdom; ${ }^{27}$ University of Liverpool and HCA Pathology Laboratories, London WC1E 6JA, United Kingdom; ${ }^{28}$ Urological Research Laboratory, Cancer Research UK Cambridge Research Institute, Cambridge CB2 ORE, United Kingdom; ${ }^{29}$ Department of Surgical Oncology, University of Cambridge, Addenbrooke's Hospital, Cambridge CB2 0QQ, United Kingdom; ${ }^{30}$ Institute of Cancer Research, Sutton, London SM2 5NG, United Kingdom; ${ }^{31}$ Department of Biological Sciences and School of Medicine, University of East Anglia, Norwich NR4 7TJ, United Kingdom; ${ }^{32}$ Division of Genetics and Epidemiology, The Institute of Cancer Research, Sutton SM2 5NG, United Kingdom; ${ }^{33}$ Royal Marsden NHS Foundation Trust, London SW3 6JJ and Sutton SM2 5PT, United Kingdom; ${ }^{34}$ Institute of Biosciences and Medical Technology (BioMediTech), University of Tampere and Tampere University Hospital, 33520 Tampere, Finland; ${ }^{35}$ University of Queensland, School of Medicine, Brisbane, QLD 4006, Australia; ${ }^{36}$ Pathology Queensland, Royal Brisbane and Women's Hospital, Brisbane, QLD 4029, Australia; ${ }^{37}$ University of Queensland, UQ Centre for Clinical Research, Brisbane, QLD 4029, Australia; ${ }^{38}$ Breast Cancer Translational Research Laboratory, Université Libre de Bruxelles, Institut Jules Bordet, 1000 Brussels, Belgium; ${ }^{39}$ Université Lyon 1, Institut National du Cancer (INCa)-Synergie, 69008 Lyon, France; ${ }^{40}$ Dana-Farber Cancer Institute, Boston, Massachusetts 02215, USA; ${ }^{41}$ Brigham and Women's Hospital, Harvard Medical School, Boston, Massachusetts 02115, USA; ${ }^{42}$ Department of Pathology, Ninewells Hospital and Medical School, Dundee DD1 9SY, United Kingdom; ${ }^{43}$ Department of Surgical Oncology, University of Texas MD Anderson Cancer Center, Houston, Texas 77030, USA

\section{Acknowledgments}

We thank Thomas Bleazard at the Faculty of Medical and Human Sciences, University of Manchester for discussion and assistance with manuscript preparation. We also thank The Cancer Genome Atlas (TCGA) Project Team and their specimen donors for providing sequencing data used in this study. This work was supported by the Wellcome Trust. Y.S.J is supported by a European Molecular Biology Organization long-term fellowship (LTF 1203_2012). J.M. C.T. is supported by Marie Curie Fellowship FP7-PEOPLE-2012IEF (project number 328264). P.J.C. is a Wellcome Trust Senior Clinical Fellow. Support was provided to A.M.F. by the National Institute for Health Research (NIHR) UCLH Biomedical Research Centre. The ICGC Breast Cancer Consortium was supported by a grant from the European Union (BASIS) and the Wellcome Trust.
The ICGC Prostate Cancer Consortium was funded by Cancer Research UK with a grant from the Dallaglio Foundation (grant number C5047/A14835). R.E. is supported by National Institute for Health Research support to the Biomedical Research Centre at The Institute of Cancer Research and Royal Marsden NHS Foundation Trust. We also thank the National Cancer Research Prostate Cancer Mechanisms of Progression and Treatment (PROMPT) collaborative (grant code G0500966/75466) which has funded tissue and urine collections in Cambridge. The authors also acknowledge financial support from the Department of Health via the National Institute for Health Research comprehensive Biomedical Research Centre award to Guy's and St. Thomas' NHS Foundation Trust and Breakthrough Breast Cancer Research (ICGC 08/09 and KCL) (A.T.).

Author contributions: Y.S.J., J.M.C.T., W.M., P.J.C., and M.R.S. conceived the project. Y.S.J. and J.M.C.T. performed mitochondrial sequence analyses and PCR validation experiments. W.M., B.F., and F.Y. conducted cytogenetics validation experiments. Y.S.J., J. M.C.T., H.R.D., M.R., Y.L., L.Y., G.G., P.S.T., S.B., E.P., A.F., M.G., and S.N-Z. performed bioinformatics analyses. J.N., A.R.G., C.C., A.B., A.T., M.T.M.L., L.J.v.V., B.K.T.T., S.A., P.N.S., J.W.M.M., S. K., A.V-S., A-L.B-D., J.E.E., A.M.F., C.F., D.E.N., C.C., R.E., S.R.L., C.D., G.T., A.L.R., C.A.P., and A.M.T. provided clinical samples and commented on the manuscript. U.M. and S.N-Z. provided conceptual advice. Y.S.J and M.R.S wrote the paper with contributions from all authors.

\section{References}

Adams KL, Palmer JD. 2003. Evolution of mitochondrial gene content: gene loss and transfer to the nucleus. Mol Phylogenet Evol 29: 380-395.

Andrews RM, Kubacka I, Chinnery PF, Lightowlers RN, Turnbull DM, Howell N. 1999. Reanalysis and revision of the Cambridge reference sequence for human mitochondrial DNA. Nat Genet 23: 147.

Baca SC, Prandi D, Lawrence MS, Mosquera JM, Romanel A, Drier Y, Park K, Kitabayashi N, MacDonald TY, Ghandi M, et al. 2013. Punctuated evolution of prostate cancer genomes. Cell 153: 666-677.

Bailey JA, Gu Z, Clark RA, Reinert K, Samonte RV, Schwartz S, Adams MD, Myers EW, Li PW, Eichler EE. 2002. Recent segmental duplications in the human genome. Science 297: 1003-1007.

Benson G. 1999. Tandem repeats finder: a program to analyze DNA sequences. Nucleic Acids Res 27: 573-580.

Brinster RL, Chen HY, Trumbauer ME, Yagle MK, Palmiter RD. 1985. Factors affecting the efficiency of introducing foreign DNA into mice by microinjecting eggs. Proc Natl Acad Sci 82: 4438-4442.

Campbell PJ, Stephens PJ, Pleasance ED, O'Meara S, Li H, Santarius T, Stebbings LA, Leroy C, Edkins S, Hardy C, et al. 2008. Identification of somatically acquired rearrangements in cancer using genome-wide massively parallel paired-end sequencing. Nat Genet 40: 722-729.

Chen JM, Chuzhanova N, Stenson PD, Ferec C, Cooper DN. 2005. Metaanalysis of gross insertions causing human genetic disease: novel mutational mechanisms and the role of replication slippage. Hum Mutat 25: $207-221$.

Crasta K, Ganem NJ, Dagher R, Lantermann AB, Ivanova EV, Pan Y, Nezi L, Protopopov A, Chowdhury D, Pellman D. 2012. DNA breaks and chromosome pulverization from errors in mitosis. Nature 482: 53-58.

Dayama G, Emery SB, Kidd JM, Mills RE. 2014. The genomic landscape of polymorphic human nuclear mitochondrial insertions. Nucleic Acids Res 42: 12640-12649.

Drost JB, Lee WR. 1995. Biological basis of germline mutation: comparisons of spontaneous germline mutation rates among Drosophila, mouse, and human. Environ Mol Mutagen 25: 48-64.

Eiyama A, Kondo-Okamoto N, Okamoto K. 2013. Mitochondrial degradation during starvation is selective and temporally distinct from bulk autophagy in yeast. FEBS Lett 587: 1787-1792.

Forment JV, Kaidi A, Jackson SP. 2012. Chromothripsis and cancer: causes and consequences of chromosome shattering. Nat Rev Cancer 12: 663-670.

Friedman JR, Nunnari J. 2014. Mitochondrial form and function. Nature 505: 335-343.

Gherman A, Chen PE, Teslovich TM, Stankiewicz P, Withers M, Kashuk CS, Chakravarti A, Lupski JR, Cutler DJ, Katsanis N. 2007. Population 
bottlenecks as a potential major shaping force of human genome architecture. PLoS Genet 3: e119.

Goldin E, Stahl S, Cooney AM, Kaneski CR, Gupta S, Brady RO, Ellis JR, Schiffmann R. 2004. Transfer of a mitochondrial DNA fragment to MCOLN1 causes an inherited case of mucolipidosis IV. Hum Mutat 24: $460-465$.

Gray MW, Burger G, Lang BF. 1999. Mitochondrial evolution. Science 283: 1476-1481.

Gribble SM, Wiseman FK, Clayton S, Prigmore E, Langley E, Yang F, Maguire S, Fu B, Rajan D, Sheppard O, et al. 2013. Massively parallel sequencing reveals the complex structure of an irradiated human chromosome on a mouse background in the Tc1 model of Down syndrome. PLoS One 8: 660482.

Hastings PJ, Lupski JR, Rosenberg SM, Ira G. 2009. Mechanisms of change in gene copy number. Nat Rev Genet 10: 551-564.

Hazkani-Covo E, Zeller RM, Martin W. 2010. Molecular poltergeists: mitochondrial DNA copies (numts) in sequenced nuclear genomes. PLoS Genet 6: e1000834.

Higgins GC, Coughlan MT. 2014. Mitochondrial dysfunction and mitophagy: the beginning and end to diabetic nephropathy? $\mathrm{Br} J$ Pharmacol 171: $1917-1942$.

Huang CY, Ayliffe MA, Timmis JN. 2003. Direct measurement of the transfer rate of chloroplast DNA into the nucleus. Nature 422: 72-76.

Ju YS, Alexandrov LB, Gerstung M, Martincorena I, Nik-Zainal S, Ramakrishna M, Davies HR, Papaemmanuil E, Gundem G, Shlien A, et al. 2014. Origins and functional consequences of somatic mitochondrial DNA mutations in human cancer. eLife 3: e02935.

Kent WJ. 2002. BLAT—the BLAST-like alignment tool. Genome Res 12: 656-664.

Lee E, Iskow R, Yang L, Gokcumen O, Haseley P, Luquette LJ III, Lohr JG, Harris CC, Ding L, Wilson RK, et al. 2012. Landscape of somatic retrotransposition in human cancers. Science 337: 967-971.

Lenglez S, Hermand D, Decottignies A. 2010. Genome-wide mapping of nuclear mitochondrial DNA sequences links DNA replication origins to chromosomal double-strand break formation in Schizosaccharomyces pombe. Genome Res 20: 1250-1261.

Li H, Durbin R. 2009. Fast and accurate short read alignment with BurrowsWheeler transform. Bioinformatics 25: 1754-1760.

Li H, Handsaker B, Wysoker A, Fennell T, Ruan J, Homer N, Marth G, Abecasis G, Durbin R; 1000 Genome Project Data Processing Subgroup. 2009. The Sequence Alignment/Map format and SAMtools. Bioinformatics 25: 2078-2079.

Liu P, Erez A, Nagamani SC, Dhar SU, Kolodziejska KE, Dharmadhikari AV, Cooper ML, Wiszniewska J, Zhang F, Withers MA, et al. 2011. Chromosome catastrophes involve replication mechanisms generating complex genomic rearrangements. Cell 146: 889-903.

Michalet X, Ekong R, Fougerousse F, Rousseaux S, Schurra C, Hornigold N, van Slegtenhorst M, Wolfe J, Povey S, Beckmann JS, et al. 1997. Dynamic molecular combing: stretching the whole human genome for high-resolution studies. Science 277: 1518-1523.

Millar DS, Tysoe C, Lazarou LP, Pilz DT, Mohammed S, Anderson K, Chuzhanova N, Cooper DN, Butler R. 2010. An isolated case of lissencephaly caused by the insertion of a mitochondrial genome-derived DNA sequence into the $5^{\prime}$ untranslated region of the PAFAH1B1 (LIS1) gene. Hum Genomics 4: 384-393.

Mishmar D, Ruiz-Pesini E, Brandon M, Wallace DC. 2004. Mitochondrial DNA-like sequences in the nucleus (NUMTs): insights into our African origins and the mechanism of foreign DNA integration. Hum Mutat 23: $125-133$.

Nik-Zainal S, Alexandrov LB, Wedge DC, Van Loo P, Greenman CD, Raine K, Jones D, Hinton J, Marshall J, Stebbings LA, et al. 2012. Mutational processes molding the genomes of 21 breast cancers. Cell 149: 979-993.

Oeggerli M, Tian Y, Ruiz C, Wijker B, Sauter G, Obermann E, Guth U, Zlobec I, Sausbier M, Kunzelmann K, et al. 2012. Role of KCNMA1 in breast cancer. PLoS One 7: e41664.

Paternoster SF, Brockman SR, McClure RF, Remstein ED, Kurtin PJ, Dewald GW. 2002. A new method to extract nuclei from paraffin-embedded tissue to study lymphomas using interphase fluorescence in situ hybridization. Am J Pathol 160: 1967-1972.

Ricchetti M, Fairhead C, Dujon B. 1999. Mitochondrial DNA repairs doublestrand breaks in yeast chromosomes. Nature 402: 96-100.

Schneider JS, Cheng X, Zhao Q, Underbayev C, Gonzalez JP, Raveche ES, Fraidenraich D, Ivessa AS. 2014. Reversible mitochondrial DNA accumulation in nuclei of pluripotent stem cells. Stem Cells Dev 23: 2712-2719.

Shay JW, Baba T, Zhan QM, Kamimura N, Cuthbert JA. 1991. HeLaTG cells have mitochondrial DNA inserted into the c-myc oncogene. Oncogene 6: $1869-1874$

Simone D, Calabrese FM, Lang M, Gasparre G, Attimonelli M. 2011. The reference human nuclear mitochondrial sequences compilation validated and implemented on the UCSC genome browser. BMC Genomics 12: 517

Smeitink J, van den Heuvel L, DiMauro S. 2001. The genetics and pathology of oxidative phosphorylation. Nat Rev Genet 2: 342-352.

Stephens PJ, McBride DJ, Lin ML, Varela I, Pleasance ED, Simpson JT, Stebbings LA, Leroy C, Edkins S, Mudie LJ, et al. 2009. Complex landscapes of somatic rearrangement in human breast cancer genomes. Nature 462: 1005-1010.

Stephens PJ, Greenman CD, Fu B, Yang F, Bignell GR, Mudie LJ, Pleasance ED, Lau KW, Beare D, Stebbings LA, et al. 2011. Massive genomic rearrangement acquired in a single catastrophic event during cancer development. Cell 144: 27-40.

Thorsness PE, Fox TD. 1990. Escape of DNA from mitochondria to the nucleus in Saccharomyces cerevisiae. Nature 346: 376-379.

Timmis JN, Ayliffe MA, Huang CY, Martin W. 2004. Endosymbiotic gene transfer: organelle genomes forge eukaryotic chromosomes. Nat Rev Genet 5: 123-135.

Tubio JM, Li Y, Ju YS, Martincorena I, Cooke SL, Tojo M, Gundem G, Pipinikas CP, Zamora J, Raine K, et al. 2014. Mobile DNA in cancer. Extensive transduction of nonrepetitive DNA mediated by L1 retrotransposition in cancer genomes. Science 345: 1251343.

Turner C, Killoran C, Thomas NS, Rosenberg M, Chuzhanova NA, Johnston J, Kemel Y, Cooper DN, Biesecker LG. 2003. Human genetic disease caused by de novo mitochondrial-nuclear DNA transfer. Hum Genet 112: 303-309.

Yu X, Gabriel A. 1999. Patching broken chromosomes with extranuclear cellular DNA. Mol Cell 4: 873-881.

Zhang H, Bosch-Marce M, Shimoda LA, Tan YS, Baek JH, Wesley JB, Gonzalez FJ, Semenza GL. 2008. Mitochondrial autophagy is an HIF1-dependent adaptive metabolic response to hypoxia. J Biol Chem 283: 10892-10903.

Received February 3, 2015; accepted in revised form April 14, 2015. 
Genome Research 26: 291-300 (2016)

\section{Corrigendum: Model-based analyses of whole-genome data reveal a complex evolutionary history involving archaic introgression in Central African Pygmies}

PingHsun Hsieh, August E. Woerner, Jeffrey D. Wall, Joseph Lachance, Sarah A. Tishkoff, Ryan N. Gutenkunst, and Michael F. Hammer

The authors would like to correct the omission of the term "model-based" in the Abstract. The corrected sentence should read as follows:

"our inference method rejects the hypothesis that the ancestors of AMH were genetically isolated in Africa, thus providing model-based whole genome-level evidence of African archaic admixture."

In addition, they also would like to make a change in the last sentence in the Introduction, and the corrected sentence should read as follows:

"Together, our results provide a model-based whole-genome perspective on archaic introgression in Africa."

The article has already been corrected in both the PDF and full-text HTML files online.

doi: 10.1101/gr.206524.116

Genome Research 25: 814-824 (2015)

Corrigendum: Frequent somatic transfer of mitochondrial DNA into the nuclear genome of human cancer cells

Young Seok Ju, Jose M.C. Tubio, William Mifsud, Beiyuan Fu, Helen R. Davies, Manasa Ramakrishna, Yilong Li, Lucy Yates, Gunes Gundem, Patrick S. Tarpey, Sam Behjati, Elli Papaemmanuil, Sancha Martin, Anthony Fullam, Moritz Gerstung, ICGC Prostate Cancer Working Group, ICGC Bone Cancer Working Group, ICGC Breast Cancer Working Group, Jyoti Nangalia, Anthony R. Green, Carlos Caldas, Åke Borg, Andrew Tutt, Ming Ta Michael Lee, Laura J. van't Veer, Benita K.T. Tan, Samuel Aparicio, Paul N. Span, John W.M. Martens, Stian Knappskog, Anne Vincent-Salomon, Anne-Lise Børresen-Dale, Jórunn Erla Eyfjörd, Ola Myklebost, Adrienne M. Flanagan, Christopher Foster, David E. Neal, Colin Cooper, Rosalind Eeles, G. Steven Bova, Sunil R. Lakhani, Christine Desmedt, Gilles Thomas, Andrea L. Richardson, Colin A. Purdie, Alastair M. Thompson, Ultan McDermott, Fengtang Yang, Serena Nik-Zainal, Peter J. Campbell, and Michael R. Stratton

The names of two co-authors, Ola Myklebost and G. Steven Bova, were omitted from the authorship list of this article. Please note the corrected list above. The author and affiliation lists have already been corrected in both the PDF and full-text HTML files online.

doi: $10.1101 /$ gr.206557.116 


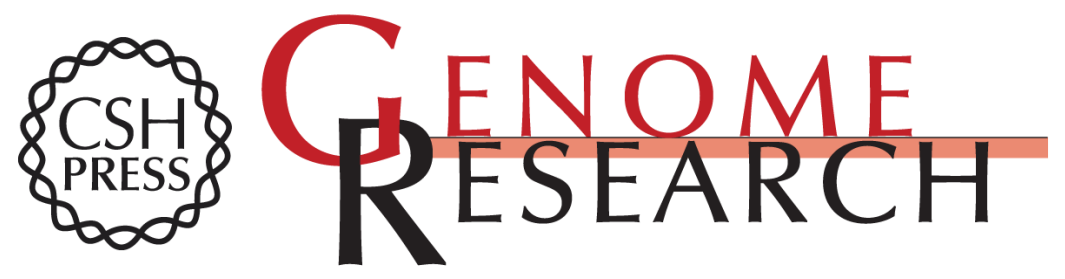

\section{Frequent somatic transfer of mitochondrial DNA into the nuclear genome of human cancer cells}

Young Seok Ju, Jose M.C. Tubio, William Mifsud, et al.

Genome Res. 2015 25: 814-824 originally published online May 11, 2015

Access the most recent version at doi:10.1101/gr.190470.115

\section{Supplemental http://genome.cshlp.org/content/suppl/2015/04/16/gr.190470.115.DC1 \\ Material}

\section{Related Content}

References

Open Access

Creative

Commons

License

Email Alerting Service
Corrigendum: Frequent somatic transfer of mitochondrial DNA into the nuclear genome of human cancer cells

Young Seok Ju, Jose M.C. Tubio, William Mifsud, et al.

Genome Res. May , 2016 26: 717.2

This article cites 48 articles, 9 of which can be accessed free at:

http://genome.cshlp.org/content/25/6/814.full.html\#ref-list-1

Articles cited in:

http://genome.cshlp.org/content/25/6/814.full.html\#related-urls

Freely available online through the Genome Research Open Access option.

This article, published in Genome Research, is available under a Creative

Commons License (Attribution 4.0 International), as described at

http://creativecommons.org/licenses/by/4.0/.

Receive free email alerts when new articles cite this article - sign up in the box at the top right corner of the article or click here.

\section{Affordable, Accurate Sequencing.}

